\title{
A Petri Method In The Management Of Public Opinion Information
}

\author{
Jin Du ${ }^{12}$, Yanhui Du ${ }^{1}, Y u$ Chen ${ }^{2}$ \\ ${ }^{1}$ Chinese People's Public Security University, Beijing \\ ${ }^{2}$ Yunnan Police Officer Academy, Kunming \\ koaladj@126.com, dyh6889@126.com, yuchen450@yahoo.com
}

\begin{abstract}
Public opinion management system plays an important role on information management nowadays. Developing characteristics and rules of online public opinion are discussed by means of Petri analyzing method in present paper. The public opinion was regarded as a 'flow' from which the conception of 'public opinion flow' was proposed and its control model was developed as well. Based on that, correlation degree variables between 'social network' cluster nodes were dynamically introduced and general rules of public opinion flows between associated network cluster organizations were studied at the time. The 'Public opinion information flow' correlation control model which can regulate the relationship between social network cluster nodes was raised and verified by means of empirical research of sociology, journalism and psychology. At last, artificial intelligence and decision support can be supplied to relevant industries by instructing system design and management system by means of the study of public opinion.
\end{abstract}

Key words: Social network cluster node; Public opinion information flow control; 'Public opinion information flow' correlation control model; Public opinion management system.

\section{Background}

With the development of emerging online media, social networking cluster also accelerate the exchange. The discovery and management of the public opinion of a focus event is challenging in information management. If some of the public opinion, the improper development of the information, it is easy to cause serious public safety and social harm. Therefore, the public opinion information management is an extremely important part of information management.

Currently, many scholars and institutions from different disciplinary perspectives and entry point for the mechanisms and laws of the events on the network public opinion research. Network public opinion information has freedom, interactivity, immediacy, occult mass characteristics, and there is a certain evolution mechanism. In order to control and guide public opinion, the proliferation of information, from the formation of public opinion information, high volatility and play down the four-stage analysis. There are many research institutions, for hotspot network public opinion information the powerful argumentation and discussion. In this paper, on the basis of the formation process of the research network public opinion, by Petri analysis method to study the law of development of the network of public opinion information. And provide a reference for the guidance and 
management to the network public opinion information.

\section{Information flow and control model of network public opinion}

Currently, there are no standard definitions of the concept of 'public opinion information flow'. According to the author, 'public opinion information flow' is a public opinion information flow between 'public opinion information node' accordance with the evolution and characteristics. Concrete expression is public opinion information exchange and delivery process for the social cluster network. On the network, the existence of consciousness insights is same or similar to social networking cluster. They identify clusters outside opinion, and introduced into the cluster. This behavior has contributed to the flow of public opinion information. In the cluster of social networking, the focus of attention is the public opinion node they held. Absorption of these public opinion judgments of the cluster makes further representations with the expression, to promote the development of public opinion. Therefore, the public opinion information flow is directional. Accordance with certain rules and principles, a member of the cluster transfer network knowledge to the recipients of public opinion information. The more obtained information of public opinion, the greater amount of information piled on. The interference factors also increase the extraction of valuable intelligence will be less. Therefore, need to maintain control and guide the intensity of moderate public opinion information. To grasp the degree of association between social networking cluster, and then control the internal flow and the flow of public opinion information. Reduce the negative public opinion information flow, and increase the flow of positive public opi- nion information. It is also in this article to study the problem.

Petri net is a language to describe the process of mathematical methods, discrete parallel system of mathematics. It invented in 1960 by Karl • A • Geoff Petrie, suitable for describing asynchronous, concurrent computer system model. A Petri net is strict mathematical formulation, intuitive graphical expression. Petri nets, formal definition of the four-tuple (library, change, input function, output function). Of any plan can be mapped to a four-tuple, and vice versa. Process modeling is a process of the state, by token modeling in place; the state change is modeled by the changes. Token represents the state of things (people, cargo, machinery), information, conditions or object; representative of the library, the channel or geographical location; Changes represents the event, transformation or transport processes (Flow), is divided into the current state can be up to state and unreachable. For public opinion, the description of the information flow, Petri nets is a suitable description of the 'flow' and concurrent tool. It has been widely used in the description of the various processes, which has good analytical ability and mathematical foundation. The formal definition of Petri nets, there are four basic elements: changes (' $\square$ '), library (' $\bigcirc$ '), arc (' $\rightarrow$ ') and logos ('• '). Its formal definition: $\mathrm{PN}=\{\mathrm{P}, \mathrm{T}, \mathrm{F}, \mathrm{M} 0\}$, the library collection $\mathrm{P}=\{\mathrm{P} 1, \ldots, \mathrm{Pn}\}$, set of transitions $\mathrm{T}=\{\mathrm{T} 1, \ldots, \mathrm{Tm}\}$, a collection of $\operatorname{arcs} \mathrm{F} \subseteq(\mathrm{P} \times \mathrm{T}) \cup(\mathrm{T} \times \mathrm{P})$, the initial identification $\mathrm{M} 0: \mathrm{P} \rightarrow\{0,1,2, \ldots\}$, that is the library $\mathrm{P}$ marking $\mathrm{M}$ initial distribution. Public opinion information flow used the formal definition of the ' $F$ ' to represent. In library collections $(\mathrm{PN}), \mathrm{PF}$ express each node as a class library. Normal distribution with the occurrence, development and outbreak of public opinion information, it is also the normal flow. Therefore the initial marking M0: $\mathrm{P}$ 
$\rightarrow\{0,1,2, \ldots\}$ to represent. When the 'flow' occurs, that is M0 state, while when the development and transformation of 'flow' were directivity, each library as P0, P1, P2, P3, ... These libraries are also public opinion information associated node in the process model. The 'flow' forward, the development and conversion also stored in the library. It according to the different social networking cluster interference and promote change.

\section{Public opinion information correlation process model}

In the cluster of social networking, the different information fusion of public opinion will have a different combination of public opinion, and even the new orientation of public opinion. In order to extract and acquire valuable intelligence of public opinion, often require extensive search for the correlation network. The same time the semantic recognition with the public opinion in the communication between network nodes to accelerate access external knowledge. For the potential implicit public opinion information, we need the valuation and analysis of filtration. Increase the flow of public opinion information search process, continuously filtering identification; reduce redundant public opinion information obtained to guide and control the flow of information in the public opinion of social networking cluster. In the stage of public opinion information generated, analysis and control of the information flow of public opinion research, can lay the foundation for further internal evolution of public opinion information about emergencies.

Based on Petri nets of public opinion information flow modeling, information exchange behavior is defined as the flow node $\mathrm{P}$ (library), $\mathrm{P}=(\mathrm{PS}, \mathrm{PT}, \mathrm{PE}$, PV).Where PS refers to a cluster of dif- ferent social networking where public opinion information flow (stream), per unit time, as the average number of units for a particular point of view, to the point of view of the number of a certain type of event; PT (type) is the type of the public opinion information, divided according to the objects produced by the public opinion information; PE (Explode) is the amount of the outbreak of the information generation of public opinion, higher than the amount of average in the unit time, once produced, the value will be presented level of the growth in the number; $\mathrm{PV}$ (velocity) is public opinion information of velocity, for the speed of the information flow model nodes defined and analyzed.

'Node' is critical of the stream of public opinion information. Public opinion Exchanged in the supply and receiving nodes. It flows in the network topology structure and social connection to the cluster. The information in the amount of occurrence of the node indicated with $\mathrm{M}$, initial value M0. These nodes create flow by the exchange of information in the model $\mathrm{T}$ (Transition). When the decentralized social clusters from different areas obtained external public opinions, they have related each other (relevance).This concept refers to the dependencies between the clusters of social networking. It is used to measure the degree of association of the cluster. The more they contact, the stronger the correlation. Therefore, the higher of value 'association (R)', the stronger dependence between nodes, and the closer contacted. Based on the above analysis, the establishment of public opinion information flow based on Petri nets associated process model:

Definition 1: seven-tuple $\Sigma=(\mathrm{P}, \mathrm{T}$, M0, S, E, PV, R) to form the PE public opinion information flow conditions:

Theorem 1: $\mathrm{PE}=\mathrm{Mt}+\operatorname{Mi}(\Delta \mathrm{t})-\mathrm{Mi}(\mathrm{t})$

$(\mathrm{P}, \mathrm{T}, \mathrm{M} 0)$ is a simple network, namely the base net of $\Sigma$.PE indicates $\mathrm{P}$ is $\mathrm{P} 1$, 
$\mathrm{P} 2, \ldots, \mathrm{Pn}, \mathrm{E}$ represents the flow rate of the public opinion information, PS represents the network social cluster has the size of the public opinion, $\mathrm{S}$ represents the scale for the opinion stream. S values can change public opinion value of the information flow model, and the speed of information flow on the node is a key factor. PV nodes on the flow rate of public opinion information, by the association between the nodes to be controlled: the higher the correlation, the public opinion information flow is simpler. R represents the correlation between nodes, the higher the correlation, the more extensive range of public opinion information flow. Thus, within a unit of time ' $\mathrm{t}$ ', the information flow by means of the node 'i' on 'Mt' and flow rate 'Mi $(\Delta t)$ ' is proportional to the growth. The significance of information modeling and control analyze useful public opinion, reducing redundant information in the public opinion information flow 'Mi (t)'.

' $t$ ' represents flow time of the units in the node; ' $\Delta \mathrm{t}$ ' is the time interval. By the node changes in the amount of information, calculate the flow rate and velocity. The control node 'i' traffic 'Mt' velocity 'Mi $(\Delta \mathrm{t})$ ' access to external media also reduce redundant information 'Mi (t)'. Public opinion information flow associated with the process, we can describe the distribution of cluster network public opinion information (including information nodes distributed topology and each node in the amount of information).

Reasoning (1) $\Delta \mathrm{Mi}(\mathrm{t})=\Sigma \mathrm{PE}_{(\mathrm{j} \rightarrow \mathrm{i})}$

Reasoning (2) $\operatorname{Mi}(\mathrm{t})=\operatorname{Mt}+\operatorname{Mi}(\Delta \mathrm{t})-\mathrm{PE}$

Reasoning (3) $\mathrm{Mi}(\mathrm{t}+1)=\mathrm{Mi}^{\prime}(\mathrm{t}) \cup \mathrm{Mi}^{\prime}{ }^{\prime}(\mathrm{t})$ $\cup \Delta \operatorname{Mi}(\mathrm{t})$

'Mi '(t) ' represents the amount of time ' $t$ ' in node ' $i$ ' outward flow of public opinion; 'Mi' '(t)' represents ' $\mathrm{t}$ ' moments in node ' $i$ ', based on the amount of different node associated with the degree of out- ward flow of public opinion; ' $\Delta \mathrm{Mi}(\mathrm{t}){ }^{\prime}$ represents ' $\mathrm{t}$ ' moment public opinion into node ' $i$ ' and generated new public opinion; 'PE $(j \rightarrow \mathrm{i})$ ' represents public opinion flows from node ' $j$ ' to node 'i' at time ' $t$ ', contains the public dissemination of information and the information generated by the correlation.

Theorem 2: $\mathrm{PVi}=(\mathrm{Mi}(\mathrm{t}+\Delta \mathrm{t})-\mathrm{Mi}(\mathrm{t})) /$ $\Delta \mathrm{t}$

$$
=\mathrm{PEi} / \Delta \mathrm{t}
$$

Reasoning (1) $P E(j \rightarrow i)=\operatorname{Mij}^{\prime}(t)+\operatorname{Rij}(t)$ $\times \operatorname{Mij}^{\prime}(\mathrm{t}) \cup \Delta \operatorname{Mij}(\mathrm{t})$

When node ' $i$ ' opinion increased rapidly, to increase the output nodes or the association between the nodes, accelerate the outflow speed of the public opinion information in a network cluster. The nodes of the cluster network ' $\mathrm{K}$ ', is the outbreak of public opinion information dissemination number tends to a finite value. Cluster webs process and access to information from different nodes in the process are dynamic.

On different nodes of the cluster network in the public opinion, the stock is different. 'I' and ' $j$ ' represents the cluster network in the organization of the i-th and $\mathrm{j}$-th, that is, the nodes in the network (where $\mathrm{i}, \mathrm{j}=1,2, \ldots \mathrm{k}$, and $\mathrm{i} \neq \mathrm{j}$ ).'Rij' associated cluster network node $i$ and node $j$ in the range $[0,1] .{ }^{\prime} \mathrm{Mj}^{\prime}$ represents the stock of public opinion on the node j.'Mij' (t) 'represents at time t nodes $\mathrm{j}$ to $\mathrm{i}$ flowing opinion.'Mij' '(t)' at time $t$ node $j$ to $i$ associated with different amount of outward flow of public opinion.' $\triangle \mathrm{Mij}(\mathrm{t})^{\prime}$ inflow from node $j$ to $i$ at time $t$ where public opinion and the generated new public opinion in the cluster.

When public opinion information into outbreak stage, especially the initial outbreak, control correlation between nodes timely, can reduce the harmful contact; then control the volume and flow of public opinion information, effectively guid- 
ance and evacuation the strength of public opinion information flow is very critical. As shown in Figure 1:



Figure $1 \mathrm{t}$ time social networking cluster node i public opinion information flow correlation control

PE indicates the direction of public opinion information flow: cluster i at time ' $\mathrm{t}$ ' flow, a transition from the left, and gradually brought together to various 'Mk'; node associated with the 'i'; identify the number of how many the amount of the node's opinion, 'Mi ( $t)^{\prime}$. PE $(j \rightarrow i)$ represents information flow from node $j$ to $i$. PV $(j \rightarrow i)$ represents a node $j$ to $i$ flowing speed. The flow and speed of the nodes in the cluster is subject to the influence of the association between the nodes. At the same time, by controlling the degree of association of the nodes, control the flow rate of inflow of public opinion; Get more the amount of information required to reduce interference. According to assumptions, to build public opinion associated with the information flow control.

Reasoning (2) (Mij '(t) + Rij (t) $\times$ Mij' '(t) $\cup \Delta \operatorname{Mij}(\mathrm{t})) / \mathrm{t}=\mathrm{PV}(\mathrm{j} \rightarrow \mathrm{i})$

Reasoning (3) $P V(j \rightarrow i)=P E(j \rightarrow i) / t$

Control of public opinion information flow, the actual sources of public opinion information, the type, quantity and bias control, manage social cluster network public opinion information. Reasonable guide public opinion information transfer research conducted. Association model, node $\mathrm{k}$ is regarded as one of the original cluster outbreak of public opinion information to other nodes as associated with other social networking cluster. They have been the development and exchange, the formation of a network of interconnected structure. Especially the outbreak of public opinion information, various public opinion views the collection, constantly associated continuous access to information from outside.

\section{Case Studies and Conclusions}

According to "the Chinese KCIS society public opinion information 2011 annual observation analysis", and "2011 hot social events data flow diagram", from January 1 to November 18 from 2011 statistics on the Chinese Internet, dissemination of affected populations composite indicator exceeded 15 million event, the list of 158 hot events, the average monthly up 14.9 the (March least eight, up to 24 in September); social and livelihood type up to 33 , the practical things political, anti-corruption and law-related police-related class breakdown twentythree Beijing, Guangdong, Henan, Jiangsu and Zhejiang are the top five. Proportion were: $21 \%$ of the people's livelihood, involving law involving police $10 \%$ repeated corruption $10 \%, 12 \%$ of the current political, social security $9 \%, 9 \%$ of public health, cultural science and education $9 \%$ and $8 \%$ of the international public opinion information enterprise Finance $6 \%, 6 \%$ of disasters and accidents.

According to the "2011 hot social events spread information map analysis", $72 \%$ of the events at the day immediately online exposure, $11 \%$ of the events occurred a month before they appear online. The earliest traditional source to the me- 
dia coverage of the event statistics is for an average of 2.49 days. Events from exposure to enter the long tail to quell the average time from 29.64 days( 60 hours). Take the middle value in accordance with the normal distribution method for peak $30 \mathrm{~h}$, the example simulation experiments to the "Taobao Confession Data": October 11 , a social network of 50,000 people cluster guidance of public opinion" to stick to attack large businesses to Taobao claims" ; 20 hours later, holding the opinion cluster increased to 15 million peak. Assuming initial opinion in the number of $[0,5]$ million and the outbreak, held by six different social networking cluster different initial views, respectively, accounted for $55 \%$ of the proportion of $57 \%, 59 \%$, $61 \%, 63 \%, 65 \%$; in the peak point of the $\mathrm{t}=20$, the calculated current PE eventually emerge probability $0.48,0.59,0.6,0.78,0.88,0.97$ whole was highly associated trends.

Public opinion information flow in more than $50 \%$ of a certain size, form information research for public opinion, "the rule of the majority theory" in journalism: the development of more than half of the initial comments, and eventually public opinion toward comprehensive and overall a decisive impact. Social networking clusters diversity of public opinion information presented in the initial stages of the generation and nonuniform distribution. Over time, the individual views to two directions: associated with higher individual advice approaching uncertain time within one function value of 0 ; Associated with very low opinion in the time evolution of the process, the influence function gradually reduced to zero; Ultimately, the level of public opinion trends will be infinitely close initially held by the majority of individuals. This is the social psychologists call "psychological resistance phenomenon" that "with the recipient point of view completely contrary to the spread is likely to arouse the recipient of the psychological mood of defiance phenomenon". Petri modeling is the meaning of a certain law of development of things, using analytical methods to evaluate and take appropriate measures to make adjustments.

\section{Acknowledgements}

National Natural Science Foundation of China (71173199)

\section{References}

[1] Wang Hongwei, He Yong, Petri net: a tool of visualization modeling support, Journal of Systems Engineering, 12(2), (1997) 73-78.

[2] Frederick E. Webster, Jr. and Yoram Wind. A general model for understanding organizational buying behavior. Journal of

[3] Petri C. Communication with automata. Technical Report RADC-TR65-377, Rome Air Dev .Center, New York, NY,1966.

[4] Michael K. Molloy(1982). Performance Analysis Using Stochastic Petri Nets . IEEE, Transactions On Computers, c-31(9):913-917 